\title{
Evidence that cytokines play a role in rheumatoid arthritis
}

\author{
Fionula M. Brennan ${ }^{1}$ and lain B. Mclnnes ${ }^{2}$
}

${ }^{1}$ Kennedy Institute of Rheumatology, Imperial College London, London, United Kingdom. ${ }^{2}$ Centre for Rheumatic Diseases, Division of Immunology, Infection and Inflammation, University of Glasgow, Glasgow, United Kingdom.

\begin{abstract}
A large number of cytokines are active in the joints of patients with rheumatoid arthritis (RA). It is now clear that these cytokines play a fundamental role in the processes that cause inflammation, articular destruction, and the comorbidities associated with RA. Following the success of TNF- $\alpha$ blockade as a treatment for RA, other cytokines now offer alternative targets for therapeutic intervention or might be useful as predictive biomarkers of disease. In this Review, we discuss the biologic contribution and therapeutic potential of the major cytokine families to RA pathology, focusing on molecules contained within the TNF- $\alpha$, IL-1, IL-6, IL-23, and IL-2 families.
\end{abstract}

\section{Introduction}

$\mathrm{RA}$ is a chronic autoimmune disease with $1 \%$ prevalence in the industrialized world. It comprises a syndrome of pain, stiffness, and symmetrical synovitis (inflammation of the synovial membrane) of diarthrodial joints (freely moveable joints such as the knee) that leads to articular destruction, functional decline, and substantial comorbidity in the cardiovascular, neurologic, and metabolic systems. Therapeutic approaches used previously relied on disease-modifying antirheumatic drugs (DMARDs) such as methotrexate and sulfasalazine that had only partial clinical benefit and were associated with significant toxicity. More recently, biologic therapeutics have revolutionized treatment and have arisen as a consequence of studies aimed at understanding the critical effector pathways operating in the disease. Extensive genetic and pathogenetic studies indicate dysregulation in both innate and adaptive immune compartments. These lead to an elaboration of autoantibody responses and dyslipidemia, which might predate clinical disease onset by up to a decade. Transition occurs thereafter to articular localization via mechanisms as yet unknown, and this leads to chronic synovitis.

RA synovial membrane contains activated B and T cells, sometimes organized into germinal center-like structures, plasma cells, mast cells, and particularly activated macrophages, all recruited via an intense neovascularization process with associated lymphangiogenesis. It is also recognized that host tissue cells (activated synovial fibroblasts, chondrocytes, and osteoclasts) are involved, mediating cartilage and bone destruction as well as feeding back to promote perpetuation of inflammation. The recruitment, activation, and effector function of each of these contributor lineages is directed principally by a network of cytokines (Figure 1).

\section{The role of cytokines in RA}

The appreciation of the role played by cytokines in RA pathogenesis reflects (arguably) the most comprehensive analysis of pathologic cytokine function in a chronic inflammatory disease

Nonstandard abbreviations used: APRIL, a proliferation-inducing ligand; BLyS, $B$ lymphocyte stimulator; $\gamma_{c}$, common $\gamma$-chain; CIA, collagen-induced arthritis; DMARD, disease-modifying antirheumatic drug; FLS, fibroblast-like synoviocyte; IL-1Ra, IL-1 receptor antagonist; LT- $\beta$, lymphotoxin- $\beta$.

Conflict of interest: The authors have declared that no conflict of interest exists. Citation for this article: J. Clin. Invest. 118:3537-3545 (2008). doi:10.1172/JCI36389. in recent years (reviewed in refs. 1-3). The availability of diseased tissue from the pathogenic site (synovial joint) has both facilitated the investigation and enabled identification of the role of key molecules involved in the pathogenesis of this disease. In this Review, we provide a historical perspective outlining those studies that identified the pivotal role of TNF- $\alpha$ in the pathogenesis of RA, leading to the first clinical trials of a biological therapeutic in this disease. Thereafter, looking forward, we address other cytokines that might play a role in the disease, including those contained in the IL-1, IL-6, and IL-23 superfamilies (as members of these cytokine superfamilies are the ones with most information available), together with selected cytokines that bind a receptor containing the common $\gamma$-chain $\left(\gamma_{c}\right)$. Importantly, the nature of rheumatoid disease has changed since both authors started their studies more than 20 years ago. This resulted in part from more aggressive intervention initiated earlier and is reflected in improved functional outcomes and reduced erosive progression manifest in fewer arthroplasties (joint replacements). Importantly, RA synovial tissue that is now obtained for ex vivo analysis is generally less cellular and inflammatory than previously analyzed tissue (authors' unpublished observations). Furthermore, because it is obtained from joint replacement surgery from "end-stage" disease, it might not be useful for identifying factors important in the early phases of disease. These facts have implications for identifying novel targets in this disease and might also contribute to the differences recently observed between results of studies in vitro and in mouse models and those on human diseased tissue. Nevertheless, we propose that novel therapeutic targets and further improvement in outcomes might be offered by continued elucidation of the effector biology of cytokines.

\section{TNF- $\alpha$ and structurally related cytokines}

TNF- $\alpha$ was identified in 1975 as the factor in serum isolated from endotoxin-treated mice that induced necrosis of a methylcholanthrene-induced murine sarcoma (4). It soon became apparent that TNF- $\alpha$ had other effects, including the ability to induce signs and symptoms of shock and multiorgan damage (5) via proinflammatory effects on vascular endothelium (reviewed in ref. 6). The demonstration that TNF- $\alpha$ played a key role in RA followed from the demonstration of its potential to degrade cartilage (7) and bone (8) in vitro. Moreover, it was shown using dissociated RA synovial mononuclear cell cultures that TNF- $\alpha$ and several 


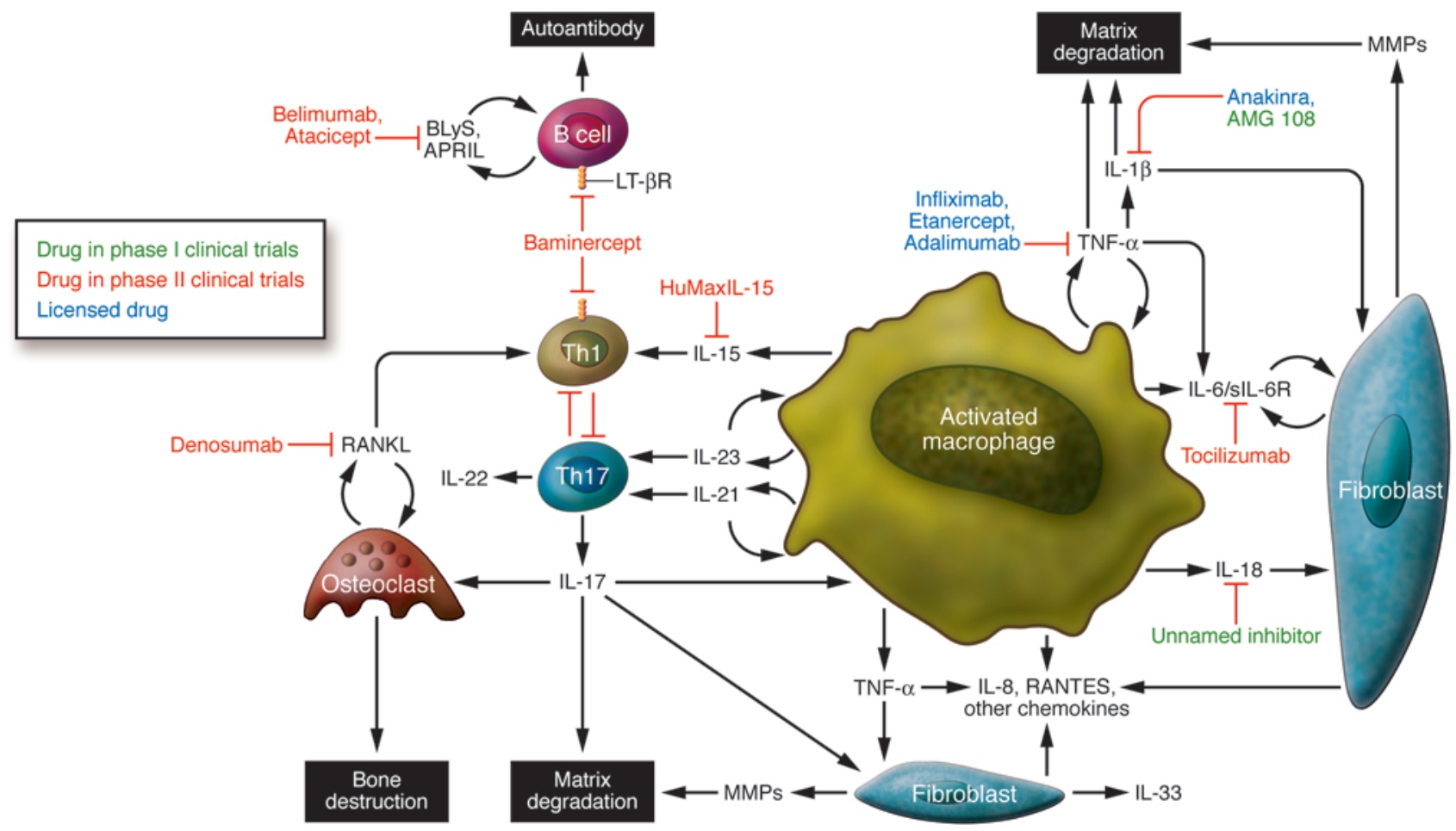

Figure 1

Cytokine targets in RA. This figure summarizes the cellular interactions believed to be of importance in the pathogenesis of RA and describes the interaction among macrophages, T cells, B cells, and nonhematopoietic cells (fibroblasts, connective tissue cells, and bone). These interactions are facilitated by the actions of cytokines released from the activated cells that then, through both autocrine (feedback on same cell) and paracrine (via other cell types) mechanisms, induce the production of other proinflammatory cytokines, which together contribute to the pathogenesis of this disease. Based on ex vivo studies from diseased tissue and in vivo studies on animal models, those cytokines with pathogenic potential have been identified and biological therapies developed to block their action. This figure identifies those therapeutic modalities and the stage in clinical development that these interventions have reached. sIL-6R, soluble IL-6 receptor.

other proinflammatory cytokines, including IL-1 (9), IL-6 (10), GM-CSF (11), and IL-8 (12), were spontaneously and chronically produced over a five-day culture period $(13,14)$. Importantly, if TNF- $\alpha$ bioactivity was blocked in these cultures, the spontaneous production of both IL-1 protein and IL1B mRNA was markedly reduced and IL-1 bioactivity was neutralized (13). This suggested that the presence of many of these cytokines was not random, but that a network or hierarchy controlled their expression. Consistent with this idea, it was subsequently shown that blockade of TNF- $\alpha$ also inhibited the spontaneous production of GM-CSF (11) (which is important for the induction and maintenance of MHC class II expression on APCs in the synovial fluid and tissue) and the expression of the proinflammatory cytokine IL- 6 and the chemokine IL-8 (15). The pathogenic effects of TNF- $\alpha$ relevant to RA disease are illustrated in Figure 2.

It was soon shown, using immunohistochemistry, that TNF- $\alpha$ and receptors for TNF- $\alpha$ (TNFRs) $(16,17)$ were expressed in human rheumatoid joint tissue and, using the collagen-induced arthritis (CIA) model of RA, that administration of an mAb specific for mouse TNF- $\alpha$ after disease onset ameliorated both inflammation and joint damage (18). Separately, Kollias and colleagues found that transgenic mice expressing the modified human TNFA gene (with replacement of the 5'-UTR regulatory sequences) spontaneously developed peripheral arthritis. This arthritis was characterized by increased human TNF- $\alpha$ protein, joint inflammation, bone erosion, and cartilage destruction (all hallmarks of RA), and disease could be ameliorated with antibodies specific for human, but not mouse, TNF- $\alpha$ (19). Together these data provided the rationale for developing therapeutics that block TNF- $\alpha$.

In 1992, the first open-label trial of a TNF- $\alpha$ blocking agent was initiated at the Kennedy Institute of Rheumatology Division, United Kingdom; 20 patients with active RA were treated with infliximab (Remicade), a chimeric antibody specific for human TNF- $\alpha$. Treatment with infliximab substantially reduced the signs and symptoms of disease, levels of C-reactive protein (CRP) in the serum, and the erythrocyte sedimentation rate (ESR) (20). This result was confirmed in other multicenter placebo-controlled trials, together with the observation that therapeutic efficacy was enhanced when infliximab was coadministered with methotrexate. This led eventually to FDA approval of the drug for the treatment of RA $(21,22)$. Importantly a subsequent two-year trial indicated that this therapy led to retardation or arrest of both joint space narrowing and bone erosion (23). In addition to infliximab, two other drugs that function as TNF- $\alpha$ blockers are licensed: etanercept (Enbrel), which is a fusion protein comprising human soluble TNFR linked to the Fc component of human IgG1, and adalimumab (Humira), which is a fully human antibody specific for human TNF- $\alpha$ (Table 1$)$.

TNF- $\alpha$ is now recognized as mediating a wide variety of effector functions relevant to the pathogenesis of RA, including endothelial cell activation and chemokine amplification, leading to leukocyte 


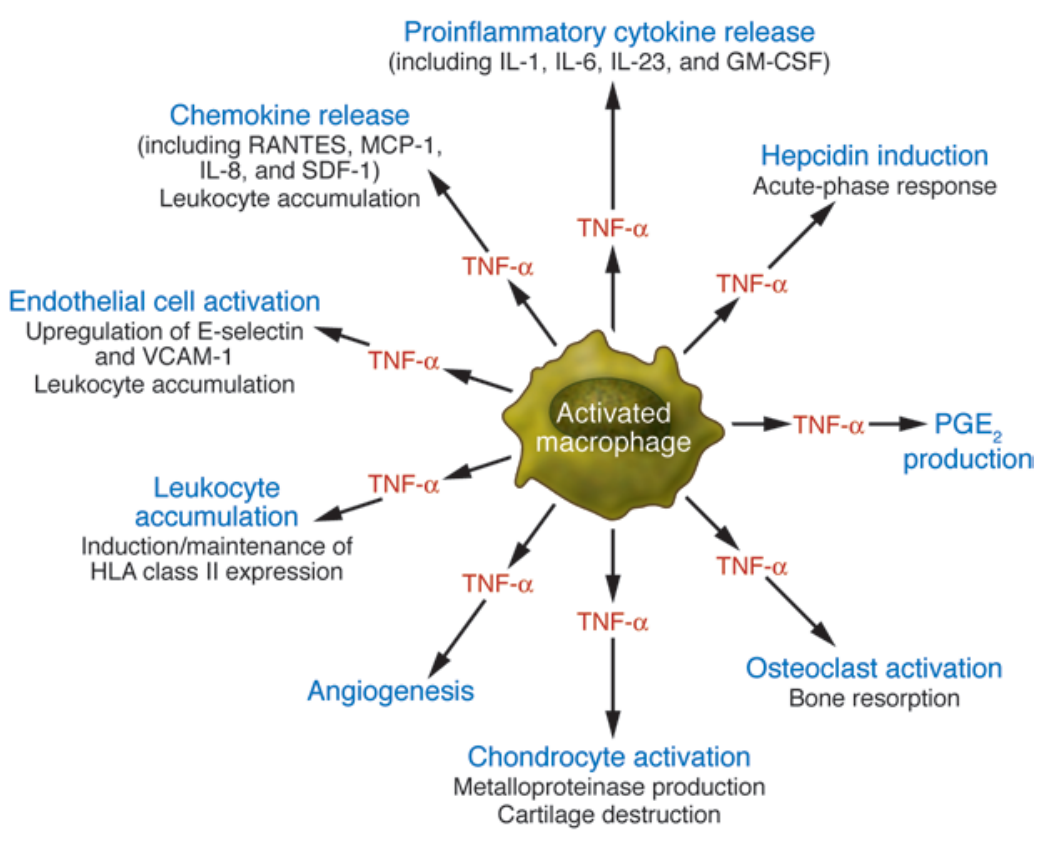

\section{Figure 2}

TNF- $\alpha$ actions relevant to the pathogenesis of RA. This figure illustrates the biological actions of TNF- $\alpha$, which is produced mainly by activated macrophages in the inflamed synovial membrane tissue in patients with RA. The actions of TNF- $\alpha$ perceived to be important in the pathogenesis of RA include its ability to induce the production of other (equally) proinflammatory cytokines, including IL-1 and IL-6, together with its ability to induce the production and release of chemokines that attract leukocytes from the blood into the inflamed tissue. This process is facilitated by upregulation of key integrins and adhesion molecules, including E-selectin and VCAM-1, on endothelium. Finally, the destruction of the underlying articular cartilage and subchondral bone is initiated by the induction of proteolytic and metalloproteinase enzymes, which bring about the destruction of the underlying cartilage, and the initiation of osteoclast resorptive activity, which brings about the destruction of the subchondral bone. SDF-1, stromal cell-derived factor-1. accumulation (24) and probably attendant cardiovascular comorbidity (25); osteoclast and chondrocyte activation, promoting articular destruction; nociceptor sensitization; impaired cognitive function; and metabolic syndrome (26). These are all recognized components of the RA disease spectrum and explain the broad effects of TNF- $\alpha$ blockade in patients. Further, therapies targeting TNF- $\alpha$ are now also recognized to be effective in multiple other chronic inflammatory diseases, including juvenile RA (JRA), Crohn disease, psoriasis, psoriatic arthritis, and ankylosing spondylitis (reviewed in refs. 24, 27).

More recently, attention has turned to the utility of additional members of the TNF superfamily as therapeutic targets (Table 1). Lymphotoxin- $\beta$ (LT- $\beta$ ) is detected in synovial membrane cells (of note, LT- $\beta$ is a cell-associated molecule rather than being secreted from the cell), and its known effector functions, particularly its role in supporting the formation of germinal centers and higher lymphoid organization, suggest that it has a role in the pathogenesis of RA (28). LT $\alpha 1 \beta 2$ and ligand for herpesvirus entry mediator (LIGHT) are both ligands of the receptor for LT- $\beta$ and have been implicated in the progression of RA. Consistent with a role for LT- $\beta$ receptor signaling, treatment of individuals with RA with an inhibitor of these two ligands, baminercept (a fusion protein comprising the extracellular domain of the human LT- $\beta$ receptor linked to the Fc component of human IgG1) resulted in significant clinical improvement in a proof-of-concept study (29). Other members of the TNF superfamily regulate B cell biology in RA. B lymphocyte stimulator (BLyS; also known as BAFF) and a proliferation-inducing ligand (APRIL) derived from fibroblast-like synoviocytes (FLSs) and mononuclear lineages in the synovium regulate $\mathrm{B}$ cell maturation, differentiation, and activation (30) and are thought likely to promote the production of autoantibodies, including rheumatoid factor and antibodies specific for cyclic citrullinated peptide. In a randomized, placebo-controlled phase I clinical trial, treatment of individuals with RA with an inhibitor of BLyS and APRIL, atacicept (a fusion protein comprising the extracellular portion of human transmembrane activator and CAML interactor [TACI], to which both BLyS and APRIL bind, linked to the Fc component of human $\operatorname{IgG1}$ ) was well tolerated, with preliminary effects on autoantibody expression noted (31). However, results of a clinical trial in RA with belimumab (an mAb that binds BLyS but not APRIL) were less impressive (32).

Finally, there is intense interest in modulating the RANKL/ RANK/osteoprotegerin (OPG) pathway. RANKL is expressed by mesenchymal cells such as FLSs and activated synovial T cells in either its membrane-bound or soluble form. It is upregulated in rodent models of RA and in RA synovium. Together with M-CSF, RANKL has a key role in osteoclast formation through its regulation of osteoclast differentiation, maturation, and induction of resorptive activity (reviewed in ref. 33). Expression of RANKL is regulated by TNF- $\alpha$ as well as other inflammatory cytokines and noncytokine mediators such as $\mathrm{PGE}_{2}$ (34). RANKL effector function is modulated by OPG, a soluble decoy receptor also expressed by mesenchymal cells that is present at increased levels in RA synovium (reviewed in ref. 35). Clinical trials assessing the effects of neutralizing RANKL using a fully human RANKL-specific mAb (denosumab) are ongoing in osteoporosis, metastatic bone disease, and RA, and the phase II clinical trials in RA seem promising with respect to suppressing erosive progression (36). Together, these data indicate that the TNF superfamily represents a rich source of therapeutic targets with future potential in the treatment of RA.

\section{IL-1 and the IL-1 superfamily members IL-18 and IL-33}

As with the TNF superfamily, several members of the IL-1 superfamily have been implicated in the pathogenesis of RA. Paradoxically, this has not as yet provided powerful therapeutics. IL- $1 \alpha$ and IL-1 $\beta$ as well as the natural IL-1 receptor antagonist (IL-1 ra) are expressed in abundance in the synovial membrane (37). IL-1 $\alpha$ and IL-1 $\beta$ in vitro induce cytokine production by synovial mononuclear cells, prostanoid and MMP release by fibroblasts, catabolism and cytokine production by chondrocytes, and bone erosion by osteoclasts (reviewed in ref. 37). Targeting IL-1 and components of the receptor for IL-1 in various rodent models of arthritis is effective in reducing inflammation and particularly articular damage $(38,39)$, and when mice engineered to express human TNF- $\alpha$ were 
Table 1

Biological therapeutics that target cytokines in RA

\begin{tabular}{|c|c|c|c|c|}
\hline Target & Name of therapeutic & Product description & Sponsor & Stage of development \\
\hline \multirow[t]{3}{*}{ TNF- $\alpha$} & Infliximab (Remicade) & Chimeric mAb specific for TNF- $\alpha$ & Centocor & Licensed \\
\hline & Etanercept (Enbrel) & Soluble p75 TNFR-human IgG1Fc fusion protein & Amgen & Licensed \\
\hline & Adalimumab (Humira) & Humanized mAb specific for TNF- $\alpha$ & Abbot & Licensed \\
\hline IL-1R & Anakinra (Kineret) & $\mathrm{IL}-1 \mathrm{ra}$ & Amgen & Licensed \\
\hline $\mathrm{IL}-1 \beta$ & AMG 108 & Humanized mAb specific for IL-1 $\beta$ & Amgen & Phase II clinical trials ${ }^{A}$ \\
\hline IL-6R & Tocilizumab (Actemra) & Humanized mAb specific for IL-6R & Roche and Chugai & Phase III clinical trials ${ }^{\mathrm{A}}$ \\
\hline IL-15 & HuMax IL-15 (AMG 714) & Humanized mAb specific for IL-15 & Genmab and Amgen & Phase II clinical trials ${ }^{A}$ \\
\hline LT $\alpha 1 \beta 2$ and LIGHT & Baminercept (BG9924) & $\begin{array}{l}\text { Extracellular domain of LT- } \beta \text { receptor- } \\
\text { human IgG1Fc fusion protein }\end{array}$ & Biogen Idec & Phase II clinical trials ${ }^{\mathrm{A}}$ \\
\hline BLyS & Belimumab (LymphoStat-B) & Humanized mAb specific for BLyS & $\begin{array}{l}\text { Human Genome Sciences } \\
\text { and GlaxoSmithKline }\end{array}$ & Phase II clinical trials ${ }^{\mathrm{A}}$ \\
\hline BLyS and APRIL & Atacicept & $\begin{array}{l}\text { Extracellular portion of TACI-human } \\
\text { IgG1Fc fusion protein }\end{array}$ & $\begin{array}{l}\text { EMD Serono and } \\
\text { ZymoGenetics }\end{array}$ & Phase II clinical trials ${ }^{A}$ \\
\hline RANKL & Denosumab (AMG162) & Humanized mAb specific for RANKL & Amgen & Phase II clinical trials ${ }^{A}$ \\
\hline
\end{tabular}

IgG1Fc, Fc portion of IgG1; IL-6R, $\alpha$-chain of the receptor for IL-6; LIGHT, ligand for herpesvirus entry mediator; LT- $\beta$ R, LT- $\beta$ receptor; TNFR, TNF- $\alpha$ receptor. ${ }^{A} F u r t h e r$ information about ongoing clinical trials can be found at http://clinicaltrials.gov.

crossed with IL-1-deficient mice, although synovial inflammation still occurred, significantly reduced bone erosion and osteoclast formation was observed (40). Further, IL-1ra-deficient mice develop spontaneous arthritis, mediated in part through amplification of Th17-dependent inflammation (41). Together these data led to the notion that IL-1 blockade would be advantageous in the context of RA, particularly with regard to articular damage. Thus far, however, clinical outcomes have been disappointing. Anakinra (a recombinant, nonglycosylated version of human IL-1 ra) reduces measures of inflammation and suppresses bone erosion in patients with RA but does so with reduced magnitude compared with agents that block TNF- $\alpha$ (42). Newer approaches to IL-1 neutralization, including antibodies specific for IL-1, IL-1 TRAP (IL-1 receptor:Fc fusion), and antibodies specific for the receptor for IL-1 remain in clinical trial (Figure 1 and Table 1). Until the outcomes of such studies are known, the long-term utility of targeting this pathway for the treatment of RA remains unclear.

IL-18 (another member of the IL-1 superfamily) is detected in RA synovium (reviewed in ref. 43), its blockade in rodent models of RA using neutralizing antibody or IL-18-binding protein delivery (a soluble IL-18R that blocks IL-18 function) is effective, and IL-18-deficient mice exhibit ameliorated CIA (44, 45). IL-18 is currently being targeted in phase I clinical trials (46). Recently, interest has arisen in IL-32 (originally known as NK4), which was discovered as an IL-18-inducible inflammatory cytokine in epithelial tissues (47). IL-32 $\gamma$ (one of the four splice variants of IL32) is expressed by synovial macrophages and promotes $\mathrm{PGE}_{2}$ and cytokine synthesis in vitro (48). In vivo, IL-32 induces TNF- $\alpha$ dependent articular inflammation but TNF- $\alpha$-independent matrix degradation (49). Notably, synovial expression correlates with local TNF- $\alpha$ and IL- $1 \beta$ levels and with ESR, suggesting a role in the pathogenesis of RA (48).

Recently, IL-33 (also known as IL-1F11) has been identified as a novel IL-1-like cytokine on the basis of its structural and functional similarities to other IL-1 family members (50). We recently detected IL-33 expression in RA synovial membrane, predominantly in FLSs (51). IL-33 administration exacerbates CIA in mice, and mice lacking IL-33R (previously known as ST2L) as well as mice administered sST2 (a natural antagonist of IL-33) exhibit reduced disease (51). Adoptive transfer experiments show that many of these effects are driven via mast cells, providing a cytokine-mediated link between FLSs and mast cells in synovitis. Additional members of the IL-1 superfamily, including IL-1F5 through IL-1F10, remain under investigation in the context of RA, although their precise biology is as yet unclear (reviewed in ref. 52).

\section{IL-6}

IL-6 was identified in 1986 as a B cell regulatory factor and is now recognized as mediating pleiotropic functions; these include effects on the maturation and activation of $\mathrm{B}$ and $\mathrm{T}$ cells, macrophages, osteoclasts, chondrocytes, and endothelial cells and broad effects on hematopoiesis in the bone marrow (reviewed in ref. 3). It is likely the primary driver of the hepatic acute phase response in RA and is implicated directly in the anemia of chronic disease through induction of hepcidin. Il6 deletion protects DBA/ 1 mice from CIA (3), and neutralization of IL- 6 using antibodies specific for either the cytokine or the $\alpha$-chain of its receptor (IL-6R) ameliorates disease (53). IL-6 signals via both chains of its heterodimeric receptor, which is composed of gp130 and IL-6R, and the signaling pathways initiated by both receptor components offer therapeutic utility (54). Pioneering studies targeting IL-6R were conducted by Nishimoto and colleagues and demonstrated significant suppression of inflammation and clinical disease activity $(55,56)$. The pivotal proof of concept for a critical role for IL-6 in the pathogenesis of RA is provided by clinical trials, now at phase III, in which tocilizumab, a humanized mAb specific for IL-6R, has been shown to suppress disease activity and erosive progression in patients with RA that is resistant to traditional DMARDs (57) (Table 1). This mAb is also useful in the treatment of systemic JRA (58) and Castleman disease (59) and is likely to offer broader utility as clinical trials expand to other diseases (reviewed in ref. 60). The toxic effects of this strategic approach remain under investigation, particularly with respect to cardiovascular safety. The place of IL-6 blockade in the therapeutic paradigm remains unclear, because the indications for which it will be licensed are pending. Efficacy in individuals with RA who fail to respond to drugs that block TNF- $\alpha$ has been demonstrated, and thus drugs blocking IL- 6 will likely provide a useful adjunct to the pharmacologic armamentarium. 
The role of other members of the IL- 6 family, including leukemia inhibitory factor (LIF) and oncostatin M, in the pathogenesis of $\mathrm{RA}$ is under ongoing investigation and might offer some therapeutic value in due course (61).

\section{IL-23 family members and their putative downstream mediator IL-17}

IL-12 is a heterodimeric cytokine, consisting of a p40 and a p35 subunit, with potent immunoregulatory properties (reviewed in ref. 62). It is released by APCs, DCs, and monocytes/macrophages in response to bacterial products and immune signals and binds a heterodimeric receptor composed of IL-12R $\beta 1$ and IL-12R $\beta 2$. It was expected that IL-12, as the main stimulator of IFN- $\gamma$ production (63) and of the development of Th1 autoimmune responses (64), would play a role in the pathogenesis of human RA. While some studies indicated a direct correlation between disease activity and elevated IL-12 in the serum and synovial fluid (65), other laboratories (including our own) did not find increased levels of functional IL-12p70 heterodimeric protein in individuals with RA.

The role of IL-12 in the pathogenesis of murine CIA is more convincing. Indeed, IL-12 can replace Mycobacterium tuberculosis when immunizing DBA/ 1 mice with type II collagen, resulting in severe arthritis that is associated with enhanced IFN- $\gamma$ production (66). Further, the severity of arthritis in this model is attenuated with antibodies specific for the p40 subunit of mouse IL-12 (67), although continued treatment in established CIA exacerbates disease (68). Therefore, IL-12 (like IFN- $\gamma$; ref. 69) has a regulatory role in mouse CIA, with the potential for an early proinflammatory and late antiinflammatory effect.

It was hypothesized subsequently that the autoimmune actions of IL-12 were in fact attributable to the newly discovered cytokine IL-23. This is also a heterodimeric cytokine composed of a unique p19 subunit and the p40 subunit component of IL-12 (70). IL-23 is secreted by activated DCs and macrophages (71) and binds to memory T cells, NK cells, macrophages, and DCs (72). Like the cytokines, the heterodimeric receptors for IL-12 and IL-23 share a component, IL-12R $\beta 1$, with IL-23R being the unique subunit of the receptor for IL-23 (73). The rationale for assuming a role for IL-23 (and not IL-12) in CIA was the observation that both mice lacking IL-23p19 (and therefore lacking IL-23) and mice lacking IL-12p40 (and therefore lacking both IL-12 and IL-23) were protected from EAE and CIA, whereas mice lacking IL-12p35 (and therefore lacking only IL-12) developed more severe disease $(72,74,75)$.

Whereas IL-12 induces the development of Th1 cells that secrete IFN- $\gamma$, IL-23 binds to memory $\mathrm{T}$ cells and induces the proliferation of a newly described subset of memory T cells, known as Th17 cells, that produce IL-17A, IL-17F, IL-6, TNF- $\alpha$, GM-CSF, and IL-22 $(76,77)$ (see below). Evidence that IL-23 might be important in human inflammatory diseases was shown in psoriasis and Crohn disease, where the p19 and p40 subunits of IL-23 were found to be expressed in affected, but not unaffected, skin (78) and inflamed intestinal tissue $(79,80)$. A recent genome-wide study showed that the gene encoding IL-23R had a highly significant association with Crohn disease and ulcerative colitis (81), although no associations with the genes encoding IL-23p19, IL-12R $\beta 1$, and IL-12p40 were identified. In contrast to animal studies $(67-69,82,83)$, there have been a limited number of studies in human arthritis. The published data indicate abundant IL-23p19 mRNA and protein but low levels of bioactive IL-23 (84-86). Further, we have recently explored the expression of IL-23 in patients with RA and also found low (but functional) levels of bioactive IL-23, although this was predominantly cell associated (P. Hillyer, M. Larche, and F.M. Brennan, unpublished observations).

The most recent addition to the IL-12 superfamily is IL-27 (87), a heterodimeric cytokine that consists of a subunit homologous to IL-12p40 (known as EBV-induced molecule 3 [EBI3]) and a novel protein, p28. IL-27 is expressed by activated monocytes/ macrophages, binds to a heterodimeric receptor composed of WSX-1 (also known as TCCR and IL-27R) and gp130, and induces the proliferation of naive T cells, promoting Th1 polarization and IFN- $\gamma$ production while suppressing the development of Th17 cells, at least in mice (88). Mice defective for WSX-1 showed increased susceptibility to EAE and had higher levels of circulating Th17 cells, indicating that IL-27 might be antiinflammatory (88). Recently, we have shown that addition of recombinant IL-27 also suppresses CIA in DBA/ 1 mice and that this is associated with a reduction in the magnitude of antigen-specific Th17 recall responses (89). However, severity of disease in EAE could be reduced by using IL-27-neutralizing antibodies, suggesting that the role of IL-27 (like that of IL-12 and IFN- $\gamma$ ) can be either pro- or antiinflammatory, depending on the phase of disease.

\section{Downstream effectors of the IL-12/IL-23 family: IL-17 and IL-22}

IL-17A is the signature cytokine of Th17 cells and has pleiotropic effects on many cell types, inducing the upregulation of NF- $\mathrm{KB}$, HLA class I, and several proinflammatory cytokines, including TNF- $\alpha$, IL-1 $\beta$, IL-6, and GM-CSF (reviewed in ref. 90). Of relevance to the pathogenesis of RA are the effects of IL-17 in driving osteoclastogenesis, leading to bone resorption $(34,90)$.

Support for the importance of IL-17A in RA was provided by the observation that neutralization of IL-17A in mice decreased the severity of antigen-induced arthritis (91). In addition, the severity of CIA is reduced in IL-17-deficient mice and mice administered IL-17-neutralizing antibodies (reviewed in ref. 92). The autoimmune arthritis that spontaneously develops in SKG mice (due to a mutation in the gene encoding ZAP-70; ref. 93) also seems to be highly dependent on $\mathrm{CD}^{+} \mathrm{T}$ cells secreting IL-17 (94). Further, this T cell-mediated autoimmune arthritis does not occur in $\mathrm{Il6}^{-/-}$or $I l 17^{-/-}$SKG mice, whereas IFN- $\gamma$ deficiency exacerbates the disease (95).

Several years ago, Miossec and colleagues described production of IL17A mRNA and IL-17A-expressing $\mathrm{CD}^{+}$cells in the sublining layer of RA synovial tissue by immunohistochemistry (96). IL-17A protein has been also been detected in RA synovial fluid and synovial membrane culture supernatants and acts in synergy with IL- $1 \beta$ and TNF- $\alpha(34,96)$. Recent reports have agreed with these observations (97), although other reports suggest that IL-17 is not so abundant $(98,99)$. Interestingly, recent work suggests that $\mathrm{CD} 4^{+} \mathrm{CD} 28^{-}$cells (reviewed in ref. 100) might act as a negative regulator of the differentiation of Th17 cells in RA. It is interesting to note that $\mathrm{CD} 4^{+} \mathrm{CD} 28^{-}$cells in synovial fluid were able to produce high levels of IFN- $\gamma$, a cytokine that is rarely found in synovial membranes (101) and has been reported to block the differentiation of Th17 cells (102). Conversely, Th17 cells negatively regulate the expansion of Th1 cells, providing a possible explanation for the paucity of IFN- $\gamma$ in RA synovial tissue.

Although these studies point to the attractiveness of IL-17 as a therapeutic target in RA, it is unclear from these studies where Th17 cell differentiation occurs and what factors are critical for 
these cells to differentiate in vivo. The cytokines required for human Th17 cell development have been the subject of much debate. There is general agreement on the factors required for the generation of mouse Th17 cells, with TGF- $\beta$ plus IL-6 (103-105) (which increases IL-23R expression and allows IL-23 to act as a survival and expansion factor) being necessary $(102,103)$. Initial studies suggested that TGF- $\beta$ was apparently not necessary for human Th17 cell development (reviewed in refs. 106, 107) and that IL-23 was sufficient to drive Th17 cell differentiation. However, more recently, TGF- $\beta$ has been viewed as necessary for Th 17 differentiation in humans (reviewed in ref. 108), and it seems that previous differences were in fact due to technical factors in the assays (serum conditions and/or contamination of the naive $\mathrm{T}$ cells with memory $\mathrm{T}$ cells).

Clinical targeting of this cytokine axis is now underway and has been focused on psoriasis. Antibody specific for the p40 subunit shared by IL-12 and IL-23 induces substantial reduction in the psoriasis index (which is used to measure the severity of the clinical symptoms of psoriasis) that is sustained beyond the predicted half-life of the antibody, suggesting a true immune modulatory effect (109). Strategies to specifically inhibit IL-23 and IL-17 are ongoing in psoriasis and in RA using $\mathrm{mAbs}$ specific for cytokineunique epitopes. Outcomes are awaited with considerable interest, since these studies will define the optimal therapeutic approach to this cytokine axis. This is particularly the case for targeting IL-17, which is not found at high levels in RA synovium, unlike other cytokines that have been targeted therapeutically, such as TNF- $\alpha$ and IL-6. There is similar interest in blockade of IL-22, which, as discussed above, is produced by Th17 cells, and these data will be critical in confirming the relative contribution of Th17 cells and their products to the pathogenesis of RA.

\section{Cytokines that bind a receptor containing $\mathrm{Y}_{\mathrm{c}}$}

IL-2 was the first cytokine shown to bind a receptor that contains $\gamma_{c}$, but this family of cytokines now includes IL-4, IL-7, IL-9, IL-15, and IL-21. Of these, particular attention has focused on IL-15 and, more recently, on IL-7 and IL-21. IL-15 is a four- $\alpha$-helix cytokine with structural similarities to IL-2; it binds a heterotrimeric receptor that contains a unique $\alpha$-chain (IL-15R $\alpha$ ), a $\beta$-chain that is also found in the receptor for IL-2, and $\gamma_{c}$. It is present in the RA synovial membrane, where it drives release of IFN- $\gamma$, IL-17, TNF- $\alpha$, and various chemokines $(110,111)$. IL-15 blockade in DBA/ 1 mice using either a modified form of human IL-15 linked to the Fc component of mouse IgG2a or a soluble fragment of the mouse $\alpha$-chain of the receptor for IL-15 reduces CIA severity $(112,113)$, whereas recombinant IL-15 can amplify disease in the absence of complete adjuvant at immunization. IL-15 has thus far been targeted in RA using a fully human IgG1 antibody specific for human IL-15 (AMG 714). In a phase I proof-of-concept study, IL-15 neutralization was well tolerated and clinical responses were observed (114). In a phase II, placebo-controlled study, similar trends toward efficacy were observed (I.B. McInnes, unpublished observations). This pathway remains under exploration in further clinical studies (Table 1).

Preclinical data are also emerging for an inflammatory role for IL-7 in RA synovitis. Thus, IL-7 is detected in low concentrations in blood (115) and the synovial membrane, and addition of IL-7 to synovial cultures is proinflammatory $(116,117)$. IL-7 amplifies the effects of interactions between $\mathrm{T}$ cells and macrophages (117), is implicated in lymphoid neogenesis, and has been shown to modify chondrocyte function $(118,119)$. It promotes osteoclastogenesis via a RANKL-dependent pathway (120). Persistent IL-7 expression has been detected in patients with RA following TNF- $\alpha$ blockade, suggesting some autonomy from TNF- $\alpha$-dependent regulation (121). IL-7 thus remains an intriguing target.

IL-21 represents another family member that is known to be present in the synovial fluid of individuals with RA and that might contribute to the pathogenesis of the disease. Thus, IL-21 mRNA and protein are detected in synovial membrane, and addition of IL-21 to synovial $\mathrm{T}$ cell cultures induces activation and cytokine release $(122,123)$. Neutralization of IL-21 in synovial cultures suppresses TNF- $\alpha$, IL-1, and IL-6 release (124) in a manner comparable to IL-15 blockade. Furthermore, in the CIA model of RA, disease was ameliorated in DBA/ 1 mice by administration of a fusion protein comprising the extracellular domain of IL-21R linked to a modified form of the Fc component of mouse IgG2a (125). These data as well as the emerging critical role for IL-21 in human Th17 cell differentiation mandate further studies of the biology of this cytokine in the context of synovitis.

\section{Concluding remarks}

RA remains a formidable clinical problem despite the remarkable advances of recent years. Progressive articular damage (radiographic progression), functional decline, and risk of comorbidities remain for a substantial proportion of patients. Crucially, there is as yet little evidence that we can reestablish immunologic tolerance and hence aim for drug-free remission on a regular basis. Drug responses and toxicities remain idiosyncratic, with few reliable biomarkers for prognostic or therapeutic purposes yet available. The foregoing discussion, although necessarily selective, has focused on cytokine activities that are either established as therapeutic targets for the treatment of RA (Figure 2 and Table 1) or offer plausible biology and encouraging preclinical and/or clinical data. We believe that they provide significant opportunity for therapeutic advancement that can directly address these unresolved clinical hurdles. Whereas cytokines are now firmly established as therapeutic targets, with blockade of TNF- $\alpha$ providing a benchmark for the development of new therapies, it is harder to define what constitutes a "good" clinical target from preclinical modeling. This reflects in part the predictive inadequacy of rodent models of RA but also a failure to fully understand the interactions that subserve cytokine networks in vivo. Since the components of these networks can now be readily measured simultaneously using multiplex or proteomics-based platforms, these issues can now be addressed directly in disease-associated tissues. Further challenges remain. Although blockade of TNF- $\alpha$ is beneficial, it is not curative and its effects are only partial, and nonresponses are common. Moreover, loss of effect has been observed in most therapeutic registries. In particular, it will now be essential to recognize that distinct cytokines most likely mediate discrete effects at different disease stages, and thus, there may be optimal targets defined by the relative disease stage of intervention. This assertion is not yet based on good evidence, and detailed studies of early disease are essential. However, the recent BeSt trial revealed that several patients with early RA receiving infliximab with methotrexate were able to stop and remain off infliximab (if they reached a low disease activity state without subsequent disease flare) (126). Remarkably, a proportion of patients remained in clinical remission for up to 4 years, with a small number (14\%) being totally drug free (i.e., not being treated with DMARDs) (126). Such early 
intervention might eventually rely not only on biologic agents, but also on small molecule entities with bioequivalent properties, which we believe will emerge in due course and change practice once again. Similarly, cytokine patterns will offer rich biomarker opportunities and will play a part in the introduction of personalized medicine strategies. Finally, and crucially, the increasing number of cytokine inhibitors now available provides an invaluable tool to define disease pathogenesis, in turn leading to rational design of novel therapeutics in years to come.

Note added in proof. After this article was accepted for publication, Biogen Idec announced in an October 9, 2008, press release that the company will discontinue development of baminercept as a therapeutic for RA because its phase II clinial trial did not meet its primary endpoint.

\section{Acknowledgments}

We thank Kathryn Bull and Gena Mellett for compiling the references.

Address correspondence to: Fionula Brennan, Kennedy Institute of Rheumatology Division, Imperial College Faculty of Medicine, 65 Aspenlea Road, London W6 8LH, United Kingdom. Phone: 44-208383-4451; Fax: 44-208-383-4496; E-mail: f.brennan@imperial.ac.uk.
1. Feldmann, M., Brennan, F.M., and Maini, R.N. 1996. Role of cytokines in rheumatoid arthritis. Annu. Rev. Immunol. 14:397-440.

2. Arend, W.P., Malyak, M., Guthridge, C.J., and Gabay, C. 1998. Interleukin-1 receptor antagonist: role in biology. Annu. Rev. Immunol. 16:27-55.

3. Kishimoto, T. 2005. Interleukin-6: from basic science to medicine -- 40 years in immunology. Annu. Rev. Immunol. 23:1-21.

4. Carswell, E.A., et al. 1975. An endotoxin-induced serum factor that causes necrosis of tumors. Proc. Natl. Acad. Sci. U. S. A. 72:3666-3670.

5. Tracey, K.J., Lowry, S.F., and Cerami, A. 1988. Cachetin/TNF-alpha in septic shock and septic adult respiratory distress syndrome. Am. Rev. Respir. Dis. 138: $1377-1379$.

6. Pober, J.S. 1988. TNF as an activator of vascular endothelium. Ann. Inst. Pasteur Immunol. 139:317-323.

7. Dayer, J.M., Beutler, B., and Cerami, A. 1985. Cachectin/tumor necrosis factor stimulates collagenase and prostaglandin E2 production by human synovial cells and dermal fibroblasts. J. Exp. Med. 162:2163-2168.

8. Bertolini, D.R., Nedwin, G.E., Bringman, T.S., Smith, D.D., and Mundy, G.R. 1986. Stimulation of bone resorption and inhibition of bone formation in vitro by human tumour necrosis factors. Nature. 319:516-518.

9. Buchan, G., et al. 1988. Detection of activated T cell products in the rheumatoid joint using cDNA probes to Interleukin-2 (IL-2) IL-2 receptor and IFN-gamma. Clin. Exp. Immunol. 71:295-301.

10. Hirano, T., et al. 1988. Excessive production of interleukin 6/B cell stimulatory factor-2 in rheumatoid arthritis. Eur. J. Immunol. 18:1797-1801.

11. Haworth, C., et al. 1991. Expression of granulocytemacrophage colony-stimulating factor in rheumatoid arthritis: regulation by tumor necrosis factoralpha. Eur. J. Immunol. 21:2575-2579.

12. Brennan, F.M., et al. 1990. Detection of interleukin 8 biological activity in synovial fluids from patients with rheumatoid arthritis and production of interleukin 8 mRNA by isolated synovial cells. Eur. J. Immunol. 20:2141-2144.

13. Brennan, F.M., Chantry, D., Jackson, A., Maini, R., and Feldmann, M. 1989. Inhibitory effect of TNF alpha antibodies on synovial cell interleukin-1 production in rheumatoid arthritis. Lancet. 2:244-247.

14. Brennan, F.M., Chantry, D., Jackson, A.M., Maini, R.N., and Feldmann, M. 1989. Cytokine production in culture by cells isolated from the synovial membrane. J. Autoimmun. 2(Suppl.):177-186.

15. Butler, D.M., Maini, R.N., Feldmann, M., and Brennan, F.M. 1995. Modulation of proinflammatory cytokine release in rheumatoid synovial membrane cell cultures. Comparison of monoclonal antiTNF $\alpha$ antibody with the IL-1 receptor antagonist. Eur. Cytokine Netw. 6:225-230.

16. Chu, C.Q., Field, M., Feldmann, M., and Maini, R.N. 1991. Localization of tumor necrosis factor $\alpha$ in synovial tissues and at the cartilage-pannus junction in patients with rheumatoid arthritis. Arthritis Rheum. 34:1125-1132.

17. Deleuran, B.W., et al. 1992. Localization of tumor necrosis factor receptors in the synovial tissue and cartilage-pannus junction in patients with rheumatoid arthritis. Implications for local actions of tumor necrosis factor alpha. Arthritis Rheum. 35:1170-1178.

18. Williams, R.O., Feldmann, M., and Maini, R.N. 1992. Anti-tumor necrosis factor ameliorates joint disease in murine collagen-induced arthritis. Proc. Natl. Acad. Sci. U. S. A. 89:9784-9788.

19. Keffer, J., et al. 1991. Transgenic mice expressing human tumour necrosis factor: a predictive genetic model of arthritis. EMBO J. 10:4025-4031.

20. Elliott, M.J., et al. 1993. Treatment of rheumatoid arthritis with chimeric monoclonal antibodies to tumor necrosis factor alpha. Arthritis Rheum. 36:1681-1690.

21. Elliott, M.J., et al. 1994. Randomised double-blind comparison of chimeric monoclonal antibody to tumour necrosis factor alpha (cA2) versus placebo in rheumatoid arthritis. Lancet. 344:1105-1110.

22. Maini, R.N., et al. 1998. Randomized placebocontrolled trial of multiple intravenous infusions of anti-TNF $\alpha$ monoclonal antibody with or without weekly methotrexate in rheumatoid arthritis. Arthritis Rheum. 41:1552-1563.

23. Lipsky, P.E., et al. 2000. 102 week clinical and radiological results from the ATTRACT trial: a 2 year, randomized, controlled, phase 3 trial of infliximab (Remicade) in patients with active rheumatoid arthritis despite methotrexate [abstract]. Arthritis Rheum. 47(Suppl.):S242.

24. Feldmann, M., and Maini, R.N. 2001. Anti-TNF $\alpha$ therapy or rheumatoid arthritis: what have we learned? Annu. Rev. Immunol. 19:163-196.

25. Dixon, W.G., and Symmons, D.P. 2007. What effects might anti-TNFalpha treatment be expected to have on cardiovascular morbidity and mortality in rheumatoid arthritis? A review of the role of TNFalpha in cardiovascular pathophysiology. Ann. Rheum. Dis. 66:1132-1136.

26. Sattar, N., McCarey, D.W., Capell, H., and McInnes, I.B. 2003. Explaining how "high-grade" systemic inflammation accelerates vascular risk in rheumatoid arthritis. Circulation. 108:2957-2963.

27. Feldmann, M., and Maini, R.N. 2003. TNF defined as a therapeutic target for rheumatoid arthritis and other autoimmune diseases. Nat. Med. 9:1245-1250.

28. Takemura, S., et al. 2001. Lymphoid neogenesis in rheumatoid synovitis. J. Immunol. 167:1072-1080.

29. Baldassare, A., et al. 2007. Preliminary safety and efficacy of LT $\beta$ R-Ig (BG9924) in the treatment of rheumatoid arthritis (RA). Presented at the American College of Rheumatology Annual Scientific Meeting. November 6-11. Boston, Massachusetts, USA. Presentation no. 947.

30. Seyler, T.M., et al. 2005. BLyS and APRIL in rheumatoid arthritis. J. Clin. Invest. 115:3083-3092.

31. Tak, P.P., et al. 2008. Atacicept in patients with rheumatoid arthritis: results of a multicenter, phase Ib, double-blind, placebo-controlled, doseescalating, single- and repeated-dose study. Arthritis Rheum. 58:61-72.

32. McKay, J., et al. 2005. Belimumab (BmAb), a fully human monoclonal antibody to B-lymphocyte stimulator (BLyS), combined with standard of care therapy reduces the signs and symptoms of rheumatoid arthritis in a heterogenous subject population [abstract]. Arthritis Rheum. 52(Suppl. 9):S710-S711.

33. Schett, G., Hayer, S., Zwerina, J., Redlich, K., and Smolen, J.S. 2005. Mechanisms of disease: the link between RANKL and arthritic bone disease. Nat. Clin. Pract. Rheumatol. 1:47-54.

34. Kotake, S., et al. 1999. IL-17 in synovial fluids from patients with rheumatoid arthritis is a potent stimulator of osteoclastogenesis. J. Clin. Invest. 103:1345-1352.

35. Horwood, N. 2008. Lymphocyte-derived cytokines in inflammatory arthritis. Autoimmunity. 41:230-238.

36. Cohen, S.B., et al. 2008. Denosumab treatment effects on structural damage, bone mineral density, and bone turnover in rheumatoid arthritis: a twelve-month, multicenter, randomized, doubleblind, placebo-controlled, phase II clinical trial. Arthritis Rheum. 58:1299-1309.

37. Dayer,J.M. 2003. The pivotal role of interleukin-1 in the clinical manifestations of rheumatoid arthritis. Rheumatology (Oxford). 42(Suppl. 2):ii3-ii10.

38. Joosten, L.A., et al. 1999. IL-1 alpha beta blockade prevents cartilage and bone destruction in murine type II collagen-induced arthritis, whereas TNFalpha blockade only ameliorates joint inflammation. J. Immunol. 163:5049-5055.

39. van den Berg, W.B., Joosten, L.A., Helsen, M., and van de Loo, F.A. 1994. Amelioration of established murine collagen-induced arthritis with anti-IL-1 treatment. Clin. Exp. Immunol. 95:237-243.

40. Zwerina, J., et al. 2007. TNF-induced structural joint damage is mediated by IL-1. Proc. Natl. Acad. Sci. U. S. A. 104:11742-11747.

41. Nakae, S., et al. 2003. IL-17 production from activated $T$ cells is required for the spontaneous development of destructive arthritis in mice deficient in IL-1 receptor antagonist. Proc. Natl. Acad. Sci. U. S. A. 100:5986-5990.

42. Furst, D.E., et al. 2005. Updated consensus statement on biological agents, specifically tumour necrosis factor $\{$ alpha\} (TNF $\{$ alpha\}) blocking agents and interleukin-1 receptor antagonist (IL1ra), for the treatment of rheumatic diseases, 2005. Ann. Rheum. Dis. 64(Suppl. 4):iv2-14.

43. McInnes, I.B., Liew, F.Y., and Gracie, J.A. 2005. Interleukin-18: a therapeutic target in rheumatoid arthritis? Arthritis Res. Ther. 7:38-41.

44. Plater-Zyberk, C., et al. 2001. Therapeutic effect of neutralizing endogenous IL-18 activity in the collagen-induced model of arthritis. J. Clin. Invest. 108:1825-1832.

45. Wei, X.Q., Leung, B.P., Arthur, H.M., McInnes, I.B., 
and Liew, F.Y. 2001. Reduced incidence and severity of collagen-induced arthritis in mice lacking IL-18. J. Immunol. 166:517-521.

46. Tak, P.P., Bacchi, M., and Bertolino, M. 2006. Pharmacokinetics of IL-18 binding protein in healthy volunteers and subjects with rheumatoid arthritis or plaque psoriasis. Eur. J. Drug Metab. Pharmacokinet. 31:109-116

47. Kim, S.H., Han, S.Y., Azam, T., Yoon, D.Y., and Dinarello, C.A. 2005. Interleukin-32: a cytokine and inducer of TNFalpha. Immunity. 22:131-142.

48. Joosten, L.A., et al. 2006. IL-32, a proinflammatory cytokine in rheumatoid arthritis. Proc. Natl. Acad. Sci. U. S. A. 103:3298-3303.

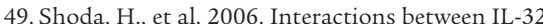
and tumor necrosis factor alpha contribute to the exacerbation of immune-inflammatory diseases. Arthritis Res. Ther. 8:R166.

50. Schmitz, J., et al. 2005. IL-33, an interleukin-1-like cytokine that signals via the IL- 1 receptor-related protein ST2 and induces T helper type 2-associated cytokines. Immunity. 23:479-490.

51. Xu, D., et al. 2008. IL-33 exacerbates antigeninduced arthritis by activating mast cells. Proc. Natl. Acad. Sci. U. S. A. 105:10913-10918.

52. Barksby, H.E., Lea, S.R., Preshaw, P.M., and Taylor, J.J. 2007. The expanding family of interleukin-1 cytokines and their role in destructive inflammatory disorders. Clin. Exp. Immunol. 149:217-225.

53. Alonzi, T., et al. 1998. Interleukin 6 is required for the development of collagen-induced arthritis. J. Exp. Med. 187:461-468.

54. Richards, P.J., et al. 2006. Functional characterization of a soluble gp130 isoform and its therapeutic capacity in an experimental model of inflammatory arthritis. Arthritis Rheum. 54:1662-1672.

55. Choy, E.H., et al. 2002. Therapeutic benefit of blocking interleukin- 6 activity with an anti-interleukin-6 receptor monoclonal antibody in rheumatoid arthritis: a randomized, double-blind, placebo-controlled, dose-escalation trial. Arthritis Rheum. 46:3143-3150.

56. Nishimoto, N., et al. 2004. Treatment of rheumatoid arthritis with humanized anti-interleukin- 6 receptor antibody: a multicenter, double-blind, placebo-controlled trial. Arthritis Rheum. 50:1761-1769.

57. Smolen, J.S., et al. 2008. Effect of interleukin-6 receptor inhibition with tocilizumab in patients with rheumatoid arthritis (OPTION study): a double-blind, placebo-controlled, randomised trial. Lancet. 371:987-997.

58. Yokota, S., et al. 2008. Efficacy and safety of tocilizumab in patients with systemic-onset juvenile idiopathic arthritis: a randomised, double-blind, placebo-controlled, withdrawal phase III trial. Lancet. 371:998-1006.

59. Nishimoto, N., et al. 2005. Humanized anti-interleukin- 6 receptor antibody treatment of multicentric Castleman disease. Blood. 106:2627-2632.

60. Nishimoto, N., and Kishimoto, T. 2006. Interleukin 6: from bench to bedside. Nat. Clin. Pract. Rheumatol. 2:619-626

61. Katoh, M. 2007. STAT3-induced WNT5A signaling loop in embryonic stem cells, adult normal tissues, chronic persistent inflammation, rheumatoid arthritis and cancer (review). Int. J. Mol. Med. 19:273-278.

62. Trinchieri, G. 2003. Interleukin-12 and the regulation of innate resistance and adaptive immunity. Nat. Rev. Immunol. 3:133-146.

63. Chan, S.H., et al. 1991. Induction of interferon gamma production by natural killer cell stimulatory factor: characterization of the responder cells and synergy with other inducers. J. Exp. Med. 173:869-879.

64. Manetti, R., et al. 1993. Natural killer cell stimulatory factor (interleukin 12 [IL-12]) induces $\mathrm{T}$ helper type 1 (Th1)-specific immune responses and inhibits the development of IL-4-producing Th cells. J. Exp. Med. 177:1199-1204.

65. Kim, W., et al. 2000. The role of IL-12 in inflammatory activity of patients with rheumatoid arthritis (RA). Clin. Exp. Immunol. 119:175-181.

66. Germann, T., et al. 1995. Administration of interleukin 12 in combination with type II collagen induces severe arthritis in DBA/ 1 mice. Proc. Natl. Acad. Sci. U. S. A. 92:4823-4827.

67. Malfait, A.M., et al. 1998. Blockade of IL-12 during the induction of collagen-induced arthritis (CIA) markedly attenuates the severity of the arthritis. Clin. Exp. Immunol. 111:377-383.

68. Joosten, L.A., Lubberts, E., Helsen, M.M., and van den Berg, W.B. 1997. Dual role of IL-12 in early and late stages of murine collagen type II arthritis. J. Immunol. 159:4094-4102.

69. Boissier, M.C., et al. 1995. Biphasic effect of interferon-gamma in murine collagen-induced arthritis. Eur. J. Immunol. 25:1184-1190.

70. Oppmann, B., et al. 2000. Novel p19 protein engages IL-12p40 to form a cytokine, IL-23, with biologi$\mathrm{cal}$ activities similar as well as distinct from IL-12. Immunity. 13:715-725.

71. Verreck, F.A., et al. 2004. Human IL-23-producing type 1 macrophages promote but IL-10-producing type 2 macrophages subvert immunity to (myco)bacteria. Proc. Natl. Acad. Sci. U. S. A. 101:4560-4565.

72. Cua, D.J., et al. 2003. Interleukin-23 rather than interleukin-12 is the critical cytokine for autoimmune inflammation of the brain. Nature. 421:744-748.

73. Parham, C., et al. 2002. A receptor for the heterodimeric cytokine IL-23 is composed of IL-12Rbeta 1 and a novel cytokine receptor subunit, IL-23R. J. Immunol. 168:5699-5708.

74. Becher, B., Durell, B.G., and Noelle, R.J. 2002. Experimental autoimmune encephalitis and inflammation in the absence of interleukin-12. J. Clin. Invest. 110:493-497.

75. Murphy, C.A., et al. 2003. Divergent pro- and antiinflammatory roles for IL-23 and IL-12 in joint autoimmune inflammation. J. Exp. Med. 198:1951-1957.

76. Aggarwal, S., Ghilardi, N., Xie, M.H., de Sauvage, F.J., and Gurney, A.L. 2003. Interleukin-23 promotes a distinct $\mathrm{CD} 4 \mathrm{~T}$ cell activation state characterized by the production of interleukin-17. J. Biol. Chem. 278:1910-1914.

77. Liang, S.C., et al. 2006. Interleukin (IL)-22 and IL-17 are coexpressed by Th17 cells and cooperatively enhance expression of antimicrobial peptides. J. Exp. Med. 203:2271-2279.

78. Lee, E., et al. 2004. Increased expression of interleukin 23 p 19 and $\mathrm{p} 40$ in lesional skin of patients with psoriasis vulgaris. J. Exp. Med. 199:125-130.

79. Fuss, I.J., et al. 2006. Both IL-12p70 and IL-23 are synthesized during active Crohn's disease and are down-regulated by treatment with anti-IL-12 p40 monoclonal antibody. Inflamm. Bowel Dis. 12:9-15.

80. Schmidt, C., et al. 2005. Expression of interleukin-12-related cytokine transcripts in inflammatory bowel disease: elevated interleukin-23p19 and interleukin-27p28 in Crohn's disease but not in ulcerative colitis. Inflamm. Bowel Dis. 11:16-23.

81. Duerr, R.H., et al. 2006. A genome-wide association study identifies IL23R as an inflammatory bowel disease gene. Science. 314:1461-1463.

82. Becher, B., Durell, B.G., and Noelle, R.J. 2003 IL-23 produced by CNS-resident cells controls T cell encephalitogenicity during the effector phase of experimental autoimmune encephalomyelitis. J. Clin. Invest. 112:1186-1191.

83. Gran, B., et al. 2002. IL-12p35-deficient mice are susceptible to experimental autoimmune encephalomyelitis: evidence for redundancy in the IL-12 system in the induction of central nervous system autoimmune demyelination. J. Immunol. 169:7104-7110.
84. Brentano, F., et al. 2008. Abundant expression of the IL-23 subunit p19, but low levels of bioactive IL-23 in the rheumatoid synovium. Ann. Rheum. Dis. Online publication ahead of print. doi:10.1136/ ard.2007.082081.

85. Kim, H.R., et al. 2007. Up-regulation of IL-23p19 expression in rheumatoid arthritis synovial fibroblasts by IL-17 through PI3-kinase-, NF-kappaBand p38 MAPK-dependent signalling pathways. Rheumatology (Oxford). 46:57-64.

86. Liu, F.L., et al. 2007. Interleukin (IL)-23 p19 expression induced by IL-1beta in human fibroblast-like synoviocytes with rheumatoid arthritis via active nuclear factor-kappaB and AP- 1 dependent pathway. Rheumatology (Oxford). 46:1266-1273.

87. Pflanz, S., et al. 2002. IL-27, a heterodimeric cytokine composed of $\mathrm{EBI} 3$ and $\mathrm{p} 28$ protein, induces proliferation of naive CD4(+) T cells. Immunity. 16:779-790.

88. Batten, M., et al. 2006. Interleukin 27 limits autoimmune encephalomyelitis by suppressing the development of interleukin 17-producing T cells. Nat. Immunol. 7:929-936.

89. Niedbala, W., et al. 2008. Interleukin-27 attenuates collagen-induced arthritis. Ann. Rheum. Dis. 67:1474-1479. doi:ard.2007.083360v1.

90. Kolls, J.K., and Linden, A. 2004. Interleukin-17 family members and inflammation. Immunity. 21:467-476.

91. Koenders, M.I., et al. 2005. Blocking of interleukin17 during reactivation of experimental arthritis prevents joint inflammation and bone erosion by decreasing RANKL and interleukin-1. Am. J. Pathol. 167:141-149.

92. Lubberts, E., Koenders, M.I., and van den Berg, W.B. 2005. The role of T-cell interleukin-17 in conducting destructive arthritis: lessons from animal models. Arthritis Res. Ther. 7:29-37.

93. Sakaguchi, N., et al. 2003. Altered thymic T-cell selection due to a mutation of the ZAP-70 gene causes autoimmune arthritis in mice. Nature. 426:454-460.

94. Hirota, K., et al. 2007. Preferential recruitment of CCR6-expressing Th17 cells to inflamed joints via CCL20 in rheumatoid arthritis and its animal model. J. Exp. Med. 204:2803-2812.

95. Hirota, K., et al. 2007. T cell self-reactivity forms a cytokine milieu for spontaneous development of IL-17+ Th cells that cause autoimmune arthritis. J. Exp. Med. 204:41-47.

96. Chabaud, M., et al. 1999. Human interleukin-17: a $\mathrm{T}$ cell-derived proinflammatory cytokine produced by the rheumatoid synovium. Arthritis Rheum. 42:963-970.

97. Lubberts, E. 2008. IL-17/Th17 targeting: on the road to prevent chronic destructive arthritis? Cytokine. 41:84-91.

98. Nistala, K., et al. 2008. Interleukin-17-producing $\mathrm{T}$ cells are enriched in the joints of children with arthritis, but have a reciprocal relationship to regulatory T cell numbers. Arthritis Rheum. 58:875-887.

99. Yamada, H., et al. 2007. Th1 but not Th17 cells predominate in the joints of patients with rheumatoid arthritis. Ann. Rheum. Dis. Online publication ahead of print. doi:10.1136/ard.2007.080341.

100.Lundy, S.K., Sarkar, S., Tesmer, L.A., and Fox, D.A. 2007. Cells of the synovium in rheumatoid arthritis. T lymphocytes. Arthritis Res. Ther. 9:202

101.Smeets, T.J., Dolhain, R.J., Breedveld, F.C., and Tak, P.P. 1998. Analysis of the cellular infiltrates and expression of cytokines in synovial tissue from patients with rheumatoid arthritis and reactive arthritis. J. Pathol. 186:75-81.

102. Harrington, L.E., et al. 2005. Interleukin 17-producing CD4+ effector $\mathrm{T}$ cells develop via a lineage distinct from the $\mathrm{T}$ helper type 1 and 2 lineages. Nat. Immunol. 6:1123-1132.

103.Veldhoen, M., Hocking, R.J., Atkins, C.J., Locksley, R.M., and Stockinger, B. 2006. TGFbeta in the con- 
text of an inflammatory cytokine milieu supports de novo differentiation of IL-17-producing T cells. Immunity. 24:179-189.

104.Bettelli, E., et al. 2006. Reciprocal developmental pathways for the generation of pathogenic effector TH17 and regulatory T cells. Nature. 441:235-238.

105.Mangan, P.R., et al. 2006. Transforming growth factor-beta induces development of the $\mathrm{T}(\mathrm{H}) 17$ lineage. Nature. 441:231-234.

106.McGeachy, M.J., and Cua, D.J. 2008. Th17 cell differentiation: the long and winding road. Immunity. 28:445-453.

107. Ouyang, W., Kolls, J.K., and Zheng, Y. 2008. The biological functions of $\mathrm{T}$ helper 17 cell effector cytokines in inflammation. Immunity. 28:454-467.

108.Yang, L., et al. 2008. IL-21 and TGF-beta are required for differentiation of human $\mathrm{T}(\mathrm{H}) 17$ cells. Nature. 454:350-352.

109. Krueger, G.G., et al. 2007. A human interleukin$12 / 23$ monoclonal antibody for the treatment of psoriasis. N. Engl. J. Med. 356:580-592.

110. McInnes, I.B., et al. 1996. The role of interleukin-15 in T-cell migration and activation in rheumatoid arthritis. Nat. Med. 2:175-182.

111. McInnes, I.B., Leung, B.P., Sturrock, R.D., Field, M., and Liew, F.Y. 1997. Interleukin-15 mediates $\mathrm{T}$ cell-dependent regulation of tumor necrosis factor-alpha production in rheumatoid arthritis. Nat. Med. 3:189-195.

112.Ferrari-Lacraz, S., et al. 2004. Targeting IL-15 receptor-bearing cells with an antagonist mutant IL-15/Fc protein prevents disease development and progression in murine collagen-induced arthritis. J. Immunol. 173:5818-5826.

113.Ruchatz, H., Leung, B.P., Wei, X.Q., McInnes, I.B., and Liew, F.Y. 1998. Soluble IL-15 receptor alphachain administration prevents murine collageninduced arthritis: a role for IL-15 in development of antigen-induced immunopathology. J. Immunol. 160:5654-5660.

114. Baslund, B., et al. 2005. Targeting interleukin-15 in patients with rheumatoid arthritis: a proof-of-concept study. Arthritis Rheum. 52:2686-2692.

115. Ponchel, F., et al. 2005. Interleukin-7 deficiency in rheumatoid arthritis: consequences for therapyinduced lymphopenia. Arthritis Res. Ther. 7:R80-R92.

116. Harada, S., et al. 1999. Production of interleukin-7 and interleukin- 15 by fibroblast-like synoviocytes from patients with rheumatoid arthritis. Arthritis Rheum. 42:1508-1516.

117.van Roon, J.A., et al. 2005. Increased intraarticular interleukin-7 in rheumatoid arthritis patients stimulates cell contact-dependent activation of CD4(+) T cells and macrophages. Arthritis Rheum. 52:1700-1710.

118.Long, D., Blake, S., Song, X.Y., Lark, M., and Loeser, R.F. 2008. Human articular chondrocytes produce IL-7 and respond to IL-7 with increased production of matrix metalloproteinase-13. Arthritis Res. Ther. 10:R23.

119.Timmer, T.C., et al. 2007. Inflammation and ectopic lymphoid structures in rheumatoid arthritis synovial tissues dissected by genomics technology: identification of the interleukin-7 signaling path- way in tissues with lymphoid neogenesis. Arthritis Rheum. 56:2492-2502.

120.Weitzmann, M.N., Cenci, S., Rifas, L., Brown, C., and Pacifici, R. 2000. Interleukin-7 stimulates osteoclast formation by up-regulating the T-cell production of soluble osteoclastogenic cytokines. Blood. 96:1873-1878.

121.van Roon, J.A., et al. 2007. Persistence of interleukin 7 activity and levels on tumour necrosis factor alpha blockade in patients with rheumatoid arthritis. Ann. Rheum. Dis. 66:664-669.

122.Jungel, A., et al. 2004. Expression of interleukin-21 receptor, but not interleukin-21, in synovial fibroblasts and synovial macrophages of patients with rheumatoid arthritis. Arthritis Rheum. 50:1468-1476.

123.Li, J., Shen, W., Kong, K., and Liu, Z. 2006. Interleukin-2 1 induces $T$-cell activation and proinflammatory cytokine secretion in rheumatoid arthritis. Scand. J. Immunol. 64:515-522.

124.Andersson, A.K., Feldmann, M., and Brennan, F.M. 2008. Neutralizing IL-21 and IL-15 inhibits proinflammatory cytokine production in rheumatoid arthritis. Scand. J. Immunol. 68:103-111.

125.Young, D.A., et al. 2007. Blockade of the interleukin-21/interleukin-21 receptor pathway ameliorates disease in animal models of rheumatoid arthritis. Arthritis Rheum. 56:1152-1163.

126.Goekoop-Ruiterman, Y.P., et al. 2008. Clinical and radiographic outcomes of four different treatment strategies in patients with early rheumatoid arthritis (the BeSt study): a randomized, controlled trial. Arthritis Rheum. 58:S126-S135. 